\title{
Automated Identification of Basic Control Charts Patterns Using Neural Networks
}

\author{
Ahmed Shaban ${ }^{1}$, Mohammed Shalaby ${ }^{2}$, Ehab Abdelhafiez ${ }^{2}$, Ashraf S. Youssef ${ }^{1}$ \\ ${ }^{1}$ Faculty of Engineering, Fayoum University, Fayoum, Egypt; ${ }^{2}$ Faculty of Engineering, Cairo University, Giza, Egypt. \\ Email: ass00@fayoum.edu.eg, mashalaby@aucegypt.edu
}

Received November $11^{\text {th }}, 2009$; revised December $5^{\text {th }}, 2009$; accepted December $10^{\text {th }}, 2009$.

\begin{abstract}
The identification of control chart patterns is very important in statistical process control. Control chart patterns are categorized as natural and unnatural. The presence of unnatural patterns means that a process is out of statistical control and there are assignable causes for process variation that should be investigated. This paper proposes an artificial neural network algorithm to identify the three basic control chart patterns; natural, shift, and trend. This identification is in addition to the traditional statistical detection of runs in data, since runs are one of the out of control situations. It is assumed that a process starts as a natural pattern and then may undergo only one out of control pattern at a time. The performance of the proposed algorithm was evaluated by measuring the probability of success in identifying the three basic patterns accurately, and comparing these results with previous research work. The comparison showed that the proposed algorithm realized better identification than others.
\end{abstract}

Keywords: Artificial Neural Networks (ANN), Control Charts, Control Charts Patterns, Statistical Process Control (SPC), Natural Pattern, Shift Pattern, Trend Pattern

\section{Introduction}

With the widespread usage of automatic data acquisition system for computer charting and analysis of manufacturing process data, there is a need to automate the analysis of process data with little or no human intervention [1]. Many researchers tried to automate the analysis of control chart patterns by developing Expert Systems to limit the human intervention in the analysis process of the control chart [2-4]. More recently; Artificial Neural Network (ANN) approach had been investigated. Dislike expert systems approaches; ANN does not require explicit rules to identify patterns. It acquires knowledge of how to identify patterns by learning. Moreover ANN models are expected to overcome the problem of high false alarm rate; because it does not depend on any statistical tests that are usually required for the traditional methods. Also, no human intervention will be required when applying ANN, and thus pattern identification can be readily integrated with inspection and rapid manufacturing technologies.

Control charts patterns are categorized as natural and unnatural patterns. The presence of an unnatural pattern such as runs, shifts in process mean, or trends as shown in Figure 1 means that a process is out of control. The accurate identification of these unnatural patterns will help the quality practitioners to determine the assignable causes for process variation; because each unnatural pattern has its related assignable causes.

Traditional control charts use only recent sample data point to determine the status of the process based on the control limits only. They do not provide any pattern related information. To increase a control chart sensitivity many supplementary rules like zone tests or run rules have been suggested by Grant and Leavenworth [5], Nelson [6], and Western Electric [7] to assist quality practitioners in detecting unnatural patterns. The primary problems with applying run rules are that the application of all the available rules simultaneously can yield an excess of false alarms due to the natural variability in the process.

This paper proposes an Artificial Neural Network algorithm to detect and identify the three basic control chart patterns; natural, shift, and trend. Natural variation is represented by normal $(0,1)$ variation, shift in process mean is expressed in terms of number of standard deviations and trend is expressed as the general slope of a trend line. This identification of each pattern is in addition to the traditional statistical detection of data runs. A run is a sequence of observations of increasing (decreas- 
ing) points or a sequence of observations above or below the process mean [8]. It is assumed that a process starts in control (has natural pattern) and then may undergo only one out of control pattern at a time (see Figure 1). For sake of simplicity only cases of upward shift and trend patterns are investigated. The proposed algorithm aims to provide a practitioner with a reliable and automated identification tool; the ANN is designed to maximize the probability of success in identifying accurately only these three basic patterns. The paper presents next a literature review, the design of the ANN network, the proposed approach for ANN, testing of the ANN algorithm and finally the performance evaluation of the algorithm.
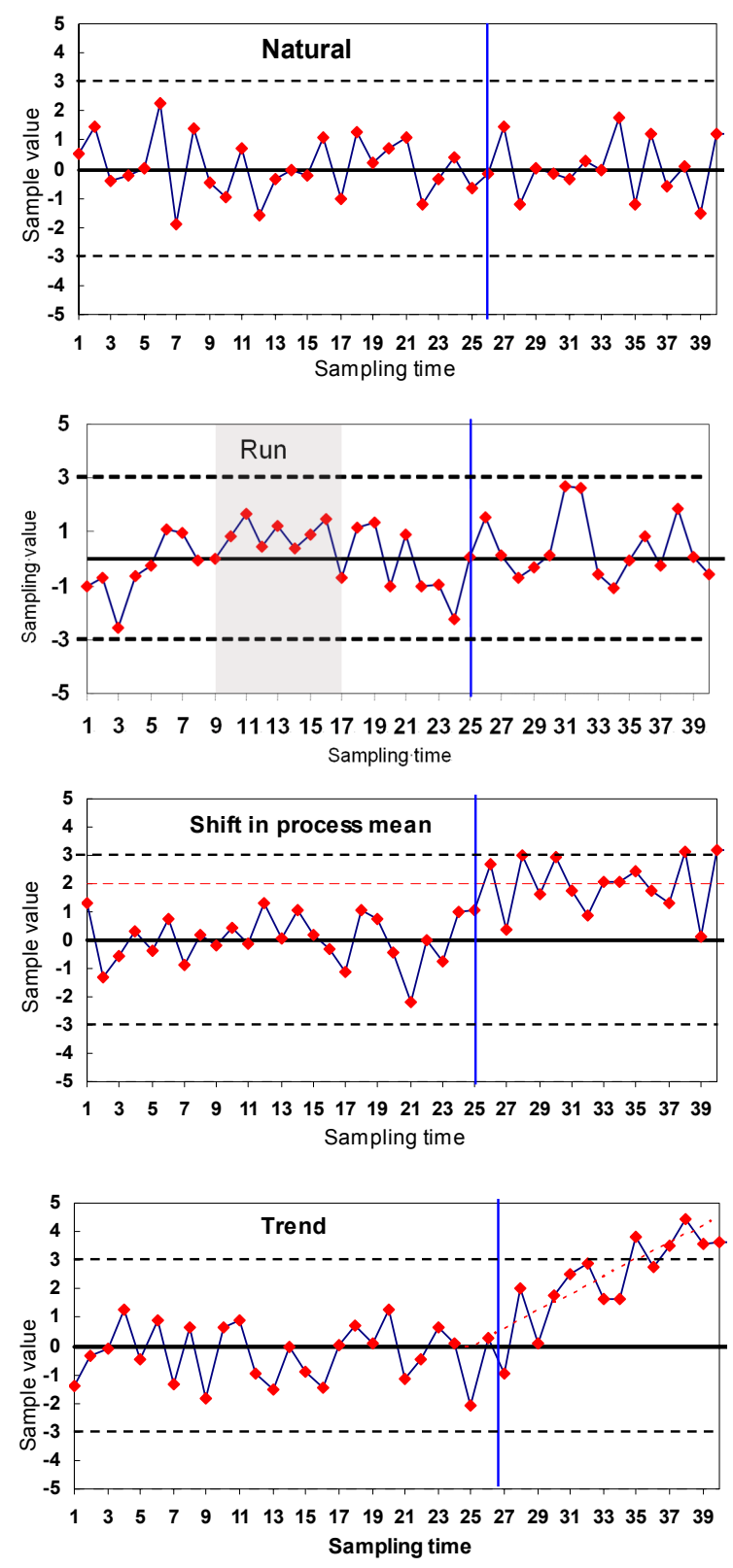

Figure 1. Basic patterns of control charts

\section{Artificial Neural Network Approaches}

ANN is investigated as an alternative tool for the traditional statistical process control tools. Some researchers tried to develop ANN models to detect sudden shifts in mean, shifts in variance, or both, and the others tried to develop ANN models to detect and identify all control chart patterns.

Smith [9] trained a single neural network to simultaneously model X-bar and $\mathrm{R}$ charts. The single output from the network was then interpreted simply as either no shift; a shift in mean; or a shift in variance. The input to smith's model was the subgroup observations plus some statistical characteristics obtained from the observations. Guo and Dooley [9] looked at positive shifts in both mean and variance using back propagation neural networks. Pugh [11,12] developed a back propagation neural network to detect a sudden shift in a process mean. Chang and Ho [13] developed a NN model that consists of two stages; stage one to detect the process variance change; and stage two to estimate the process variance magnitude. Their work resembles the R-chart function; where the R-chart signals out of control when the process variance had shifted. They extended their work and proposed an integrated neural network model to detect both a sudden process mean shift, and variance shift [14]. Also Dedeakayogullari and Burnak [15] developed two independent ANNs networks, one to detect the process mean change, and the second to detect the process variance change. The outputs of these two networks are analyzed to decide which shift has occurred. Cheng and Cheng [16] combined the traditional variance charts and ANN to detect the variance changes sources in a multivariate process. The traditional generalized variance chart works as a variance shift detector. When an outof-control signal is generated, a classifier based ANN will determine which variable is responsible for the variance shift. Chena and Wang [17] developed an artificial neural network model to supplement the multivariate $X^{2}$ chart. The method identifies the characteristic or group of characteristics that cause the signal, and also classifies the magnitude of the shifts when the $X^{2}$ statistic signals a mean shift has occurred.

Guh and Hsieh [18] proposed a neural network model to identify control chart unnatural patterns and estimate key parameters of the identified pattern. The model was intended to identify natural, upward shift, downward shift, upward trend, downward trend, and cyclic pattern. Guh [19] developed a hybrid learning-based model, which integrates ANN and decision tree learning techniques, to detect typical unnatural patterns, while identifying the major parameter (such as the shift displacement or trend slope) and the starting point of the detected pattern. The model comprises two modules in series, Module I and Module II. Module I, comprises a general-purpose system that was designed and trained to 
detect various types of unnatural patterns, and implements a procedure for classifying the actual type of the detected pattern. Module II is a special-purpose system that comprises seven specialized networks that are designed and trained to estimate the major parameters of the unnatural patterns. Similarly [20-24], and [25] utilized ANN to develop pattern recognizers that identify the abnormal control chart patterns.

Of special interest are the works of Cheng [26], Guh et al. [19], and Gauri and Chakraborty [27]. Cheng [26] developed a neural network model to detect gradual trends and sudden shifts in the process mean. The network structure was consisting of, an input layer consists of 17 nodes (neuron), hidden layer consist of 9 nodes, and output layer consist of only one node. The output node is the decision node about the presence of trend or sudden shift. His work emphasized only the detection (not the identification) of the present pattern. He evaluated his network by calculating the average run length and comparing with traditional control charts CUSUM and EWMA.

This paper will focus on the identification of the three basic patterns of control chart natural, upward shift and upward trend see Figure 1. A new neural network design will be discussed and a compatible training algorithm with the network structure will be selected. Also A new strategy to design the contents of the training data set will be introduced to minimize the required training data set size while improving the network performance.

\section{Basic Neural Network Design}

An artificial neural network consists mainly of an input layer, hidden layers, and output layer. Each layer consists of a set of processing elements or neurons, Figure 2. Figure 3 represents a single neuron with R-elements input vector. The individual element inputs $\mathrm{p}_{1}, \mathrm{p}_{2}, \mathrm{p}_{3} \ldots \mathrm{p}_{\mathrm{R}}$ are multiplied by weights $\mathrm{w}_{1,1}, \mathrm{w}_{1,2} \ldots \mathrm{w}_{1, \mathrm{R}}$ and the weighted values are fed to the summing junction. Their sum is simply $\mathrm{Wp}$, the dot product of the (single row) matrix $\mathrm{W}$ and the vector $\mathrm{p}$. The neuron has a bias $\mathrm{b}$, which is summed with the weighted inputs $\mathrm{Wp}$ to form the net input $n$. This sum, $n$, is the argument of the transfer function $\mathrm{f}$. The structure of the single neuron in Figure 3 is the same for all the neurons in the network. The network connection weights and biases are being optimized to learn the network to do its function.

The design of a network for a certain application consists of the determination of the number of hidden layers, number of neurons in each layer and the type of the transfer function where there are many types of transfer functions. The design of a suitable network is not an easy task, as there are many $\mathrm{NN}$ architectures which would satisfy an intended application [28]. Sagiroglu, Besdok and Erler [29] emphasized that no systematic method to

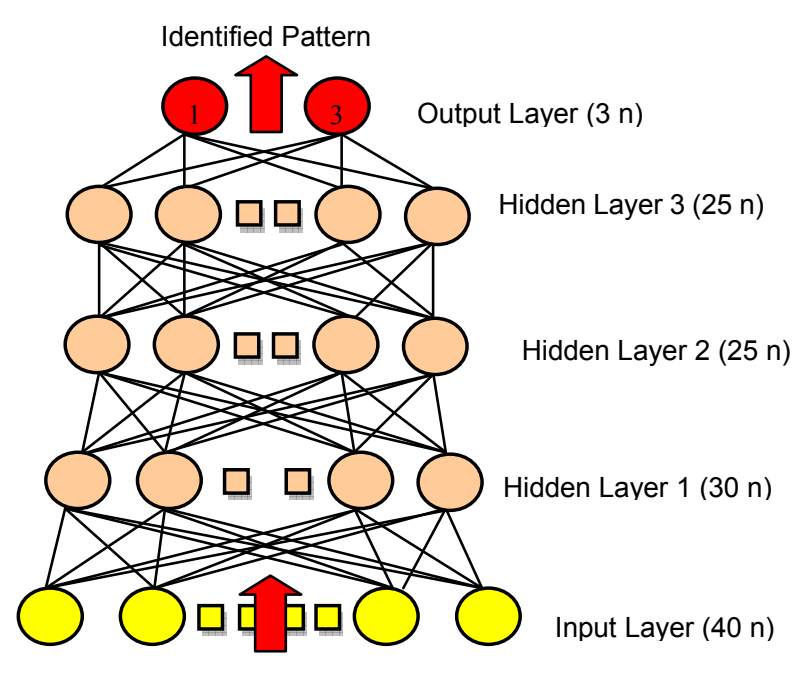

Process Data (40 points)

Figure 2. Network structure

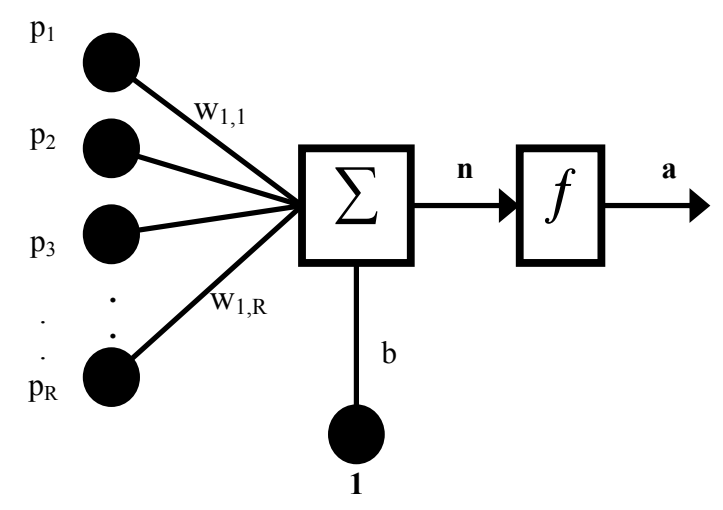

Figure 3. A single neuron [31]

select the optimum parameters. Guh [30] used the Genetic Algorithm (GA) to determine the neural networks configurations and the training parameters instead of using the trial and error method but in his latest work he used the selected parameters by trial and error method to construct his network.

In this research a Multilayer feed forward Neural Network trained with the back ropagation learning rule is adopted to develop and train the network. In the literature the number of the hidden layers ranges between 1 and 2 . Guh and Hsieh [18], Cheng [26], Gauri and Chakraborty [27] and Assaleh and Al-assaf [21] used only one hidden layer; Guh et al. [1] and Guh [19] used two hidden layers in their networks. Guh et al. [1] reported that, networks with two hidden layers performed better than those with one hidden layer. In this research 3 hidden layers were selected because this structure realized a good performance in a set of preliminary experiments. Also the size of the network is selected to be large enough to overcome the over fitting problem, one of the problems that occur 
during neural network training is called over fitting [31]. The error on the training set is driven to a very small value, but when new data is presented to the network the error is large. The network has memorized the training examples, but it has not learned to generalize to new situations. One method for improving network generalization is to use a network that is just large enough to provide an adequate fit. The larger network, the more complex the functions the network can create. Also the number of neurons in each hidden layer was selected based on a set of preliminary experiments. Guh et al. [1] also reported that, as the number of hidden neurons is increased, the learning results are usually improved too. As shown in Figure 2 the network architecture was selected to be (40-30-25-25-3). The numbers of the hidden layers were selected to be 3 , the first hidden layer consists of 30 neurons, the second and the third consists of 25 neurons each; the output layer consists of 3 neurons where each neuron is assigned for a certain pattern from the three patterns of interest.

The transfer function is an essential element in neural networks and has a great effect on their performance. In the literature the hyperbolic tangent function (tansig) and sigmoid transfer function (logsig) were adopted by many researchers in developing their networks. As shown in Figure 4 the tansig function receives an input and transfers to an output ranges between -1 and 1 , and the logsig function transfers the input to the range 0 and 1 . These two functions work well with the back propagation algorithm because they are differentiable functions and the back propagation adjusts network weights based on the MSE function's gradient which is calculated by the first partial derivatives of the MSE function. Guh et al. [1] selected the sigmoid transfer function for the hidden and output layers, but Gauri and Chakraborty [27] selected the tansig function for the hidden layers and the logsig for the output layer. Based on a set of preliminary experiments the sigmoid transfer function was selected for both the hidden and output layers of the neural network in this study.

\section{Network Training Data Generation}

Neural networks can not perform their functions without training. In this research the supervised learning approach

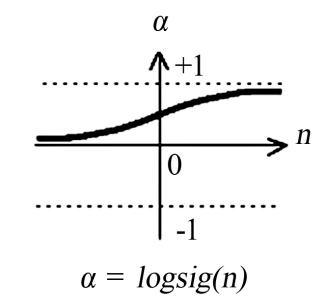

Log-Sigmoid Transfer Function

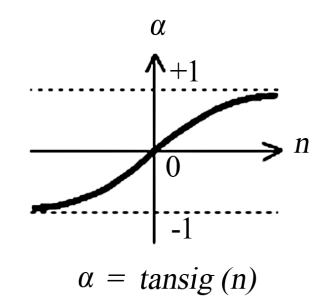

Tan-Sigmoid Transfer Function
Figure 4. Transfer functions [31] was adopted to optimize the network weights.

This approach was used by many previous researchers in this area. The training process is performed by introducing the training examples to the network; the output neuron value is compared with a target value, the difference between the target and the actual values is calculated and represented by MSE. With the aid of the training algorithm the network weights should be optimized to minimize the MSE see Figure 7. This process is repeated until a satisfactory MSE value is obtained. So that a sufficient number of training examples sets are required to train the neural networks. A Monte-Carlo simulation approach was adopted by the previous researchers to generate the training and testing data.

The Monte-Carlo simulation approach was adopted to generate the basic three control chart patterns (Figure 1) that may be exhibited by a control chart. The following equations were used to generate these patterns.

$$
\begin{array}{cc}
\text { Natural pattern } & x(t)=\mu+n(t) \sigma \\
\text { Upward shift pattern } & x(t)=\mu+n(t) \sigma+d \\
\text { Upward trend pattern } & x(t)=\mu+n(t) \sigma+s t
\end{array}
$$

In the above equations $x(t)$ is a random variable following the normal distribution and represents the sample average value at a certain time $t, \mu$ is the natural process mean, $\sigma$ is the process standard deviation, and $n(t)$ is the natural variability element in the process, and follows the normal distribution with $\mu_{n}=0$ and $\sigma_{n}=1$. The term $d$ in the shift pattern equation represents the positive shift displacement from the process in control mean. The term $s$ in the trend pattern equation represents the positive trend pattern slope. The training data was generated with $\mu=0$ and $\sigma=1$ to ensure the generality of the network for any process parameters.

In this research the identification window size consists of 40 points, or 40 sampled observations, also this size represents the size of the training examples. These 40 points actually represents the recently drawn samples from the process. This size is nearly the average of the different sizes used in the literature. In practical situations the process starts in control and then goes out of control. Cheng [26] recommended training a network with a mixture of natural and unnatural pattern to avoid high type II error. Guh et al. [1] and Guh [19] assumed that the process starts in-control and then goes out-of control in the practical situations, and generated training examples have size of 24 points include both in-control and out of control points. This strategy will allow large process change to be detected quickly by changing the start time of the different parameters of the unnatural patterns. In this study all the unnatural patterns start at the $26^{\text {th }}$ point in the training examples see Figure 5. All the shift and trend training examples were generated to have the first 25 points in control (natural pattern) and 

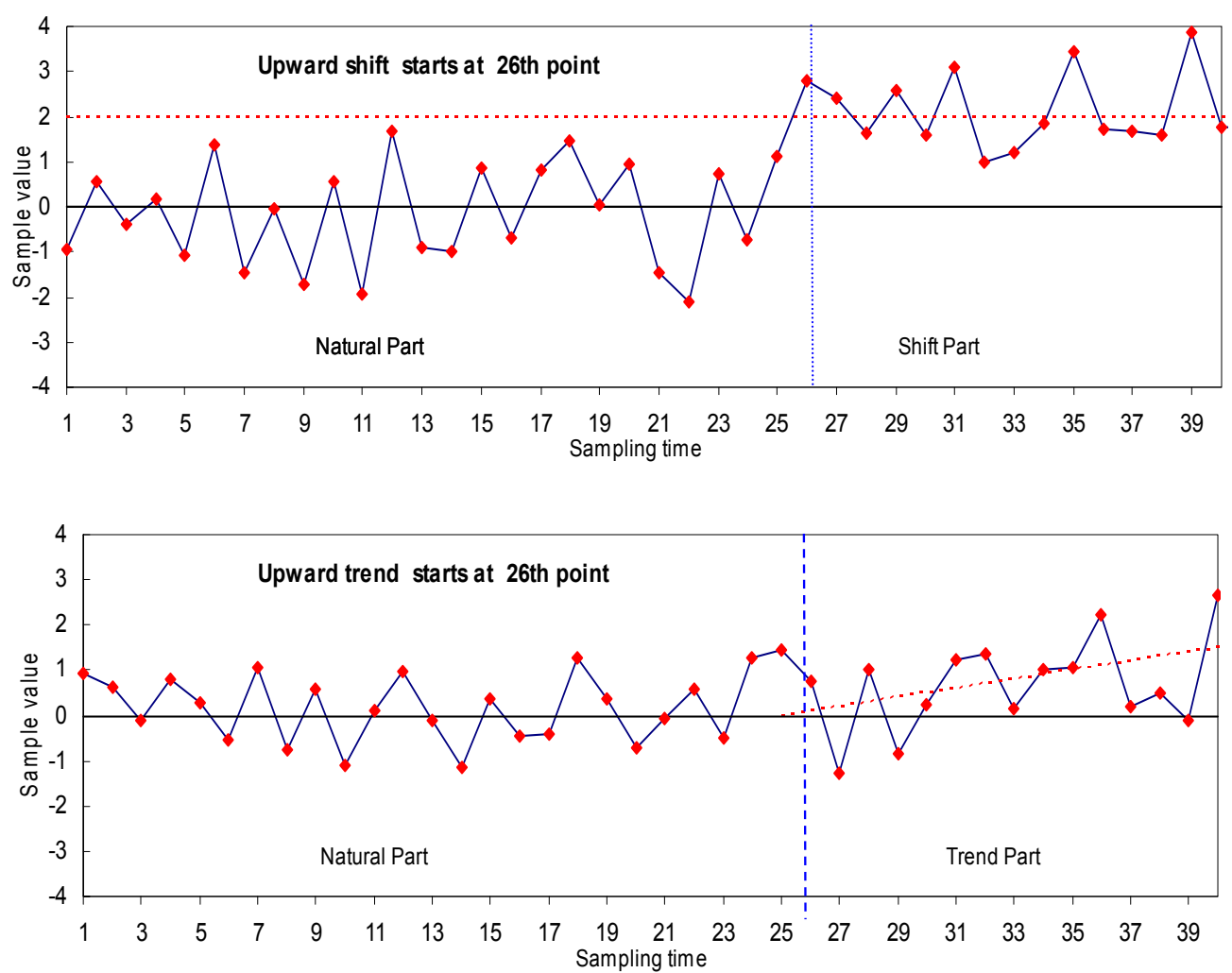

Figure 5. Two training examples

the last 15 points are out of control and contain the unnatural pattern (shift or trend) to simulate the practical situations. The flowchart of the random patterns generation routine is given in Appendix A.

The details of the training data set and their related target values for the output neurons are recorded in Table 1.

The design of the contents of the training data set is very important and has a great effect on the network performance. No particular rules to design the appropriate training data set were followed in the literature. Previous researchers were generating training examples at multiple parameters for a single pattern to cover a wide range of the pattern parameters. It was claimed that this strategy will make the network handle a general problem. Guh [19] for example, generated the shift pattern training sets to cover a range of shift magnitudes from $1 \sigma$ to $3 \sigma$. Nine training sets were generated for the shift pattern within this range; in increments of $0.25 \sigma$. Each set consisted of 90 examples. Similarly 9 training data sets were generated for the upward trend pattern, each data set consists of 90 examples. This strategy was adopted nearly by all the reviewed work in this area. Training a network with multiple patterns and multiple parameters for each pattern is expected to make the network confused and may lead to misclassifying the actual patterns to other patterns that have similar features and will also make the learning process more difficult. Based on a set of preliminary experiments, the misclassification problem appeared at a certain pattern parameters. For example, when a network was trained with trend of $0.05 \sigma$ slope, and $1 \sigma$ shift the trend pattern was misclassified as natural and vice versa. The network confusion happened because the trend slope is very small and the length of the trend pattern is also small this make the trend pattern very similar to the natural. Confusion between the shift and trend happens when training the network with multiple trend and shift patterns parameters.

A new training strategy is adopted in this study by using less training data sets for each single pattern. The injection of a certain training data sets with lesser number of parameters may help to solve this problem. In the above example, when the training data set was only supported with another trend data set having a slope of 0.1 sigma the classification accuracy was improved over a wide range of trend slopes. Table 2 presents five alternative training data sets each set contains a certain pattern parameters. After investigating these alternatives, classification accuracy improved by training the network with the lesser number parameters or with the addition of specific data set to solve a specific misclassification problem. In Table 2, Set (5) was the best alternative where it realized small MSE and excellent identification. Thus lesser 
number of pattern parameters are recommended to train the network. To train the network only one shift and two trend slope parameters were used. Preliminary experiments showed that networks trained by this way perform better over a wide range of shift and trend parameters. This approach also will minimize the required time to train the network; because it requires smaller number and sizes of training data sets. The selected structure of the training data set is Set (5). Two hundred training examples were generated for each pattern parameter within the selected data set, the sum of all the training examples is 800 which represents the size of the training data set. All sampled observations within a particular example were randomly generated from normal distribution, where a single example consists of 40 observations. All examples were filtered from runs, since runs can be easily detected by traditional computational rules without the need for the ANN algorithm. Any training example consists of 25 points of a natural pattern and the 15 points of a selected unnatural pattern. All generated observations within any example from a normal $(0,1)$ distribution will be filtered from runs.

The presence of the runs affects the identification process of the ANN badly. As shown in Figure 6(a) a run starts at the 29th point in a natural pattern training example, the run makes the series like shift, in Figure 6(b) the run makes the natural pattern like the trend pattern, in Figures 6(c, d) the shift pattern may be approximated to trend pattern. Runs could be randomly generated during the random generation of the different examples. A simple computational process for runs was applied to identify two types of runs.

1) If 5 out of 6 points are monotone increasing or decreasing;

2) If 5 out of 6 points above or below the mean value.

Once run is detected, the corresponding example is excluded from the data set. Figure 6 shows generated training examples have runs.

\section{Network Training Process}

After preparing the training data set, the network must be initialized for training. MATLAB 7 Neural Toolbox and environment were used to develop and train the network. The training patterns also were generated by MATLAB 7 generator. After initiating the network, the initial connection weights and biases were initialized by the built-in Nguyen-Widrow initialization algorithm in MATLAB [31].

Neural network is trained based on a comparison of the output and the target, until the network output matches the desired target (see Figure 7). Typically many input/target pairs are used, in this supervised learning approach to train a network. In Figure 7 the input represents the training examples of the control chart patterns, output is the obtained output neurons based on the current values of the weights and biases, and the target is the desired neurons' output. As shown in Table 1 each input pattern has its desired neuron's output, target value.

The training process is an optimization process in which the (performance or objective) function is the Mean Square Error (MSE) and the decision variables are the connection weights and biases. The target is to minimize the MSE by changing and adjusting the weights and biases to realize minimum MSE. There are many variations of the back propagation training algorithm, they adjust the network weights by making many learning cycles until the weights and biases reach their optimum values which realize the minimum MSE. In the literature the delta rule algorithm was adopted by many researchers to train their networks. In this study the Resilient Back propagation algorithm was adopted to train the network.

Table 1. Training data set structure

\begin{tabular}{|c|c|c|c|c|c|c|}
\hline \multirow{2}{*}{ Pattern } & \multirow{2}{*}{ Parameters } & \multirow{2}{*}{ Pattern start time } & \multirow{2}{*}{$\begin{array}{l}\text { Size of train- } \\
\text { ing sets }\end{array}$} & \multicolumn{3}{|c|}{ Output neurons desired output } \\
\hline & & & & neuron1 & neuron2 & neuron3 \\
\hline Natural & $\mu=0, \sigma=1$ & - & 200 & 1 & 0 & 0 \\
\hline Upward Shift & $d=1 \sigma$ & 26 & 200 & 0 & 1 & 0 \\
\hline Upward Trend & $s=0.05 \sigma, 0.1 \sigma$ & 26 & 400 & 0 & 0 & 1 \\
\hline
\end{tabular}

Table 2. Different alternatives training data sets

\begin{tabular}{cccc}
\hline \multirow{2}{*}{ Training data set } & \multicolumn{3}{c}{ Training data set structure } \\
\cline { 2 - 4 } & Natural & Shift magnitudes & Trend slopes \\
Set(1) & $\mu=0, \sigma=1$ & $1 \sigma, 2 \sigma, 3 \sigma$ & $0.05 \sigma, 0.1 \sigma, 0.3 \sigma, 0.5 \sigma$ \\
Set(2) & $\mu=0, \sigma=1$ & $2 \sigma$ & $0.1 \sigma$ \\
Set(3) & $\mu=0, \sigma=1$ & $2 \sigma$ & $0.05 \sigma, 0.1 \sigma$ \\
Set(4) & $\mu=0, \sigma=1$ & $1 \sigma$ & $0.05 \sigma$ \\
Set(5) & $\mu=0, \sigma=1$ & $1 \sigma$ & $0.05 \sigma, 0.1 \sigma$ \\
\hline
\end{tabular}

$* * \mu$ is the process mean and equal 0 ; and $\sigma$ is the process standard deviation and equal 1. 


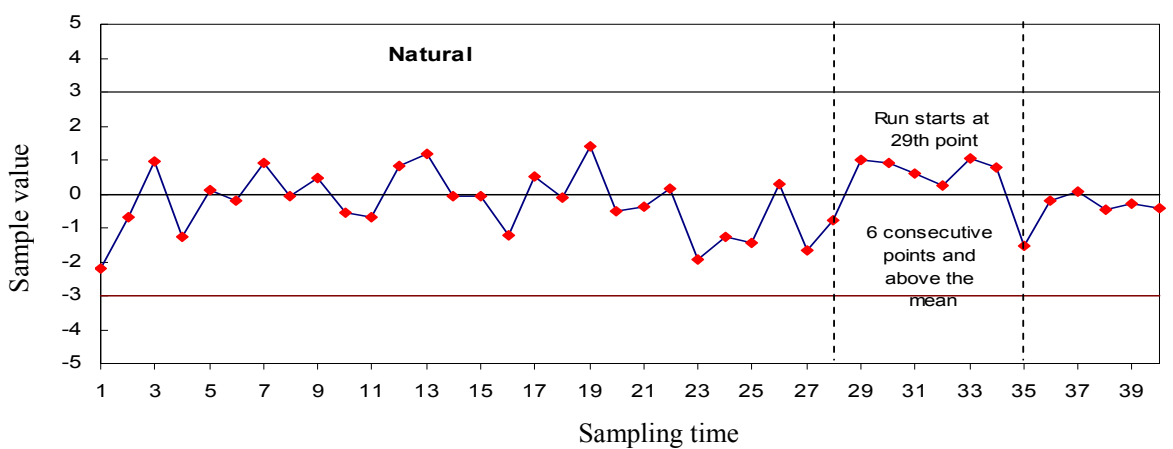

(a)

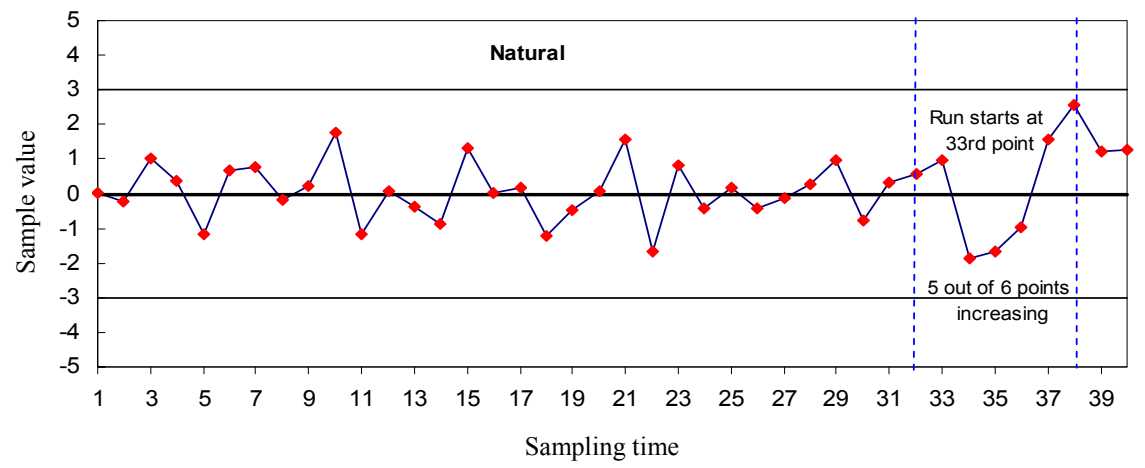

(b)

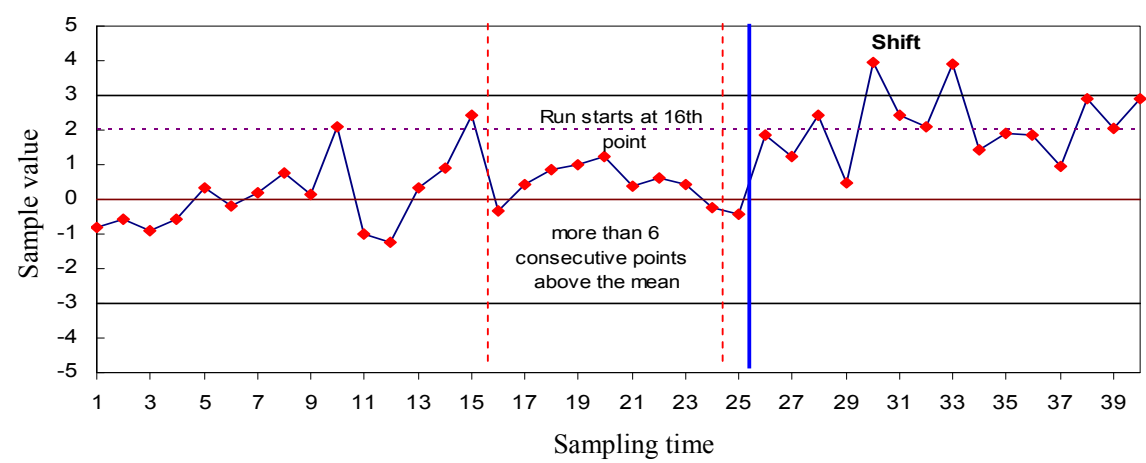

(c)

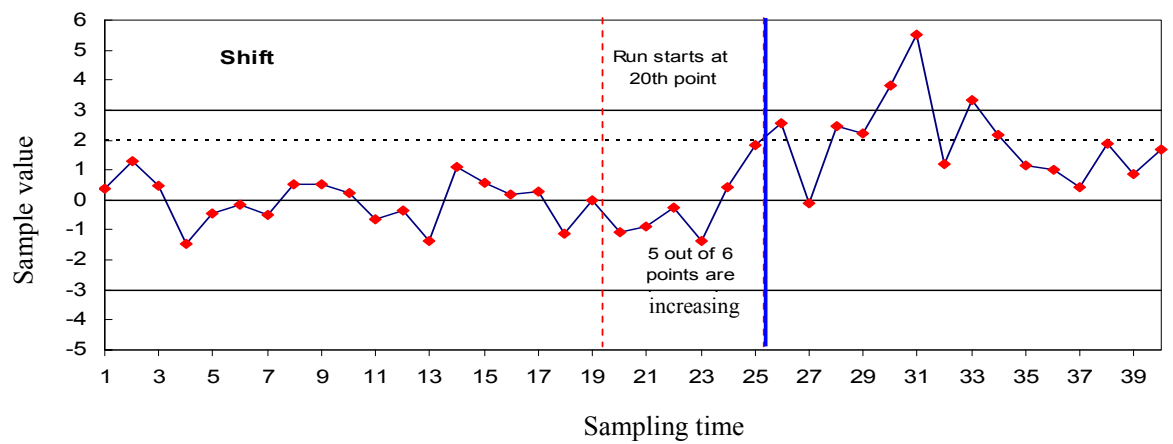

(d)

Figure 6. Training examples exhibiting runs

This algorithm was selected to be compatible with the network structure to eliminate harmful effects of the se- lected sigmoid functions. Sigmoid functions are characterized by the fact that their slopes must approach zero 


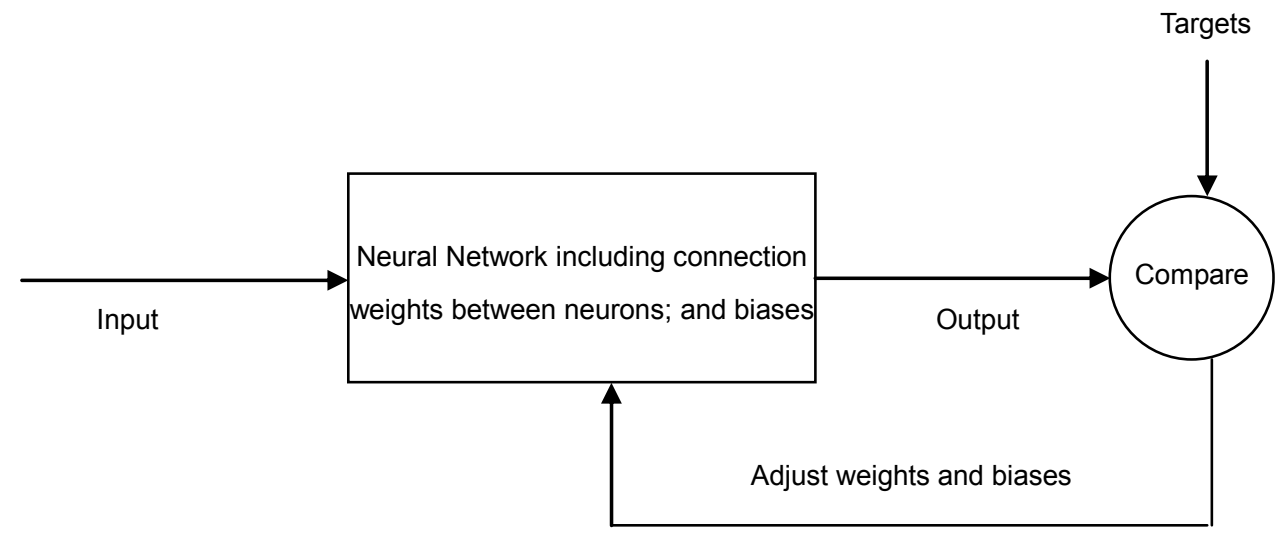

Figure 7. Supervised training

as the input gets large, this causes a problem when using steepest descent to train a multilayer network with sigmoid functions. The gradient in this case can have a very small magnitude and, therefore, cause small changes in the weights and biases, even though the weights and biases are far from their optimal values. The purpose of the resilient Back propagation training algorithm is to eliminate these harmful effects of the magnitudes of the partial derivatives. Only the sign of the derivative is used to determine the direction of the weight update; the magnitude of the derivative has no effect on the weight update.

The network training convergence condition was set to $\mathrm{MSE}=10^{-40}$ and the maximum number of learning cycles allowed to reach was set to be 100 epochs. While network is trained by these parameters, the network converged within 100 epochs as seen in Figure 8 with MSE $=2.055 \times 10^{-35}$. The small MSE value was realized by using less number of pattern parameters to train the network. After training, the network was tested by the training data set and realized $100 \%$ correct identification for all the patterns.

\section{Network Testing and Performance Evaluation}

After training the network, it must be tested and evaluated to measure its effectiveness for use. Probability of success measure was used by Guo and Dooly [10], Smith [9], Assaleh and Al-assaf [21], Guh [19], and Gauri and Chakraborty [27] to evaluate the performance of their trained neural networks. Probability of success expresses the percentage of correct identification, and it measures the capability of the network to detect and classify the pattern to the target class. In the literature the probability of success was found under different names such as the classification rate, the classification accuracy, recognition rate, and recognition accuracy. In this study the probability of success term will be used instead of the previous names. Al-assaf [32] and Guh [19] defined the

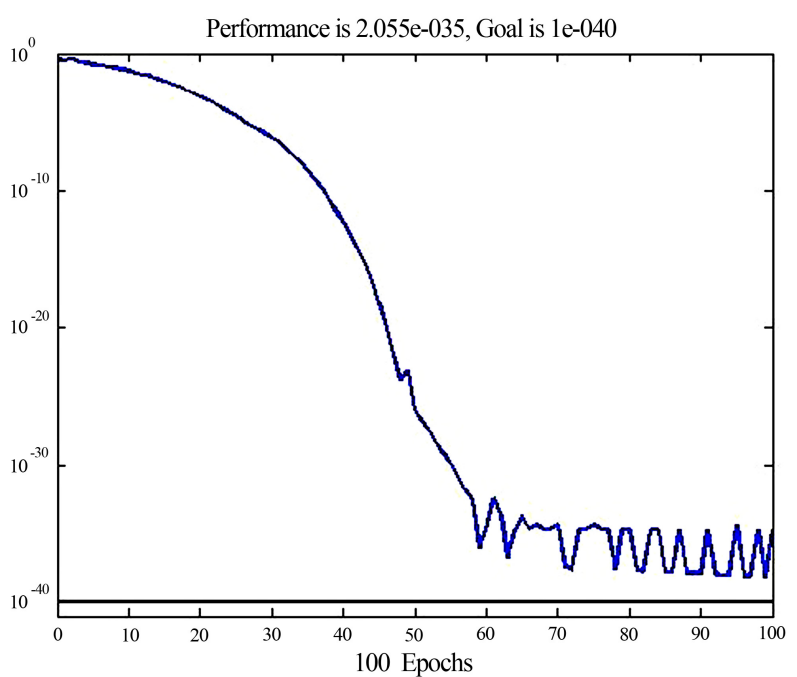

Figure 8. Training process output (MSE vs. Epoch number)

classification rate as the number of correctly recognized examples divided by total number of examples.

To measure the probability of success a new set of data was randomly generated by using the patterns equations of Section 4. The trained network is tested at multiple parameters for each pattern to assure the network generalization for different pattern parameters. A set of 200 testing example was generated for each pattern parameter. Thus, a set of 200 testing example was generated for natural pattern; a total of 600 testing example was generated for shift pattern to test the network at $1 \sigma$, $2 \sigma$, and $3 \sigma$; and a total of 800 testing example was generated for trend pattern to test the network at slopes of $0.05 \sigma, 0.1 \sigma, 0.3 \sigma$, and $0.5 \sigma$. As mentioned earlier, each example consists of 40 points; 25 points of natural pattern followed by 15 points of the tested patterns.

Table 3 exhibits the three target patterns (known by construction of the testing examples), three possible identifications patterns, and the percentage of success of the ANN to identify a given target pattern. Three pattern 
parameters were used for upward shift and 4 parameters were used for upward trend. Table entries represent the average percentages of success resulted from testing 10 different randomly generated data sets for a single given parameter. Table 4 exhibits the percentage mean, standard deviation, max and min of success of the 10 sampled data sets for each pattern parameter. The fourth column in Table 3 represents the percentage that the ANN was unable to make an identification decision. The following procedure was applied to all generated examples to obtain Table 3 results.

Step1: Input a testing example to the trained network;

Step2: Find the network output (the three values of the three output neurons $\mathrm{v}_{1}, \mathrm{v}_{2}, \mathrm{v}_{3}$ ); and find maximum output value $\mathrm{v}_{\max }$;

Step3: If $\mathrm{v}_{\max }>=0.01$, then Identify the present pattern based on $\mathrm{v}_{\max }$, if $\mathrm{v}_{\max }$ comes from the first neuron the pattern is natural; else if it comes from the second neuron the pattern is upward shift; else the pattern is upward trend;

Step4: Else if $\mathrm{v}_{\max }<0.01$, the present pattern is unknown.
Results of Table 3 show that the network can perform well in identifying the three basic patterns of control chart at a wide range of parameters. Moreover, the variation between Min and Max percentage of success for replications of the data sets is minimal which implies robustness in identification. However, a misclassification problem happened between the natural and the trend pattern that has small slope, where $1.8 \%$ of the natural testing examples were miss-classified as upward trend and $1.6 \%$ of the upward trend that has slope $0.05 \sigma$ was miss-classified as natural. The misclassification happened because the upward trend at small slopes like $0.05 \sigma$ is very similar to natural pattern; also the small pattern length in the testing examples makes the upward trend very similar to natural. Figure 9 shows two cases of similarity between natural and upward trend with slope $0.05 \sigma$.

The ANN performance is compared to the reported results in the literature. The percentage of success results are compared with Al-assaf [32], Guh [19], and Gauri and Chakraborty [27] results. Their reported results are the most recent and appear to be the highest percentage of success in identifying control chart patterns. Gauri and

Table 3. Average probability of success results based on 10 runs

\begin{tabular}{|c|c|c|c|c|c|}
\hline \multirow{2}{*}{$\begin{array}{l}\text { Target } \\
\text { Pattern }\end{array}$} & \multirow{2}{*}{$\begin{array}{c}\text { Testing } \\
\text { Parameter }\end{array}$} & \multicolumn{4}{|c|}{ ANN Identification Percentages } \\
\hline & & Natural & Upward shift & Upward trend & Unknown \\
\hline Natural & & 98.2 & 0 & 1.8 & 0 \\
\hline \multirow{4}{*}{ Upward shift } & $1 \sigma$ & 0 & 100 & 0 & 0 \\
\hline & $2 \sigma$ & 0 & 100 & 0 & 0 \\
\hline & $3 \sigma$ & 0 & 100 & 0 & 0 \\
\hline & Average & $\mathbf{0}$ & 100 & $\mathbf{0}$ & $\mathbf{0}$ \\
\hline \multirow{5}{*}{ Upward trend } & $0.05 \sigma$ & 1.6 & 0 & 98.35 & 0.05 \\
\hline & $0.1 \sigma$ & 0 & 0.15 & 99.85 & 0 \\
\hline & $0.3 \sigma$ & 0 & 0 & 100 & 0 \\
\hline & $0.5 \sigma$ & 0 & 0 & 100 & 0 \\
\hline & Average & 0.4 & 0.038 & 99.55 & 0.013 \\
\hline
\end{tabular}

Table 4. Probability of success results summary of the 10 runs

\begin{tabular}{cccccc}
\hline \multirow{2}{*}{ Target Pattern } & & \multicolumn{2}{c}{ Actual Identification } \\
\cline { 3 - 6 } & & Average & Standard deviation & Max & Min \\
\hline \multirow{2}{*}{ Natural } & $1 \sigma$ & 98.2 & 0.258 & 98.5 & 98 \\
\hline \multirow{2}{*}{ Upward shift } & $2 \sigma$ & 100 & 0 & 100 & 100 \\
& $3 \sigma$ & 100 & 0 & 100 & 100 \\
\hline \multirow{3}{*}{ Upward trend } & $0.05 \sigma$ & 98.35 & 0 & 99.5 & 97 \\
& $0.1 \sigma$ & 99.85 & 0.669 & 100 & 99.5 \\
& $0.3 \sigma$ & 100 & 0.242 & 100 & 100 \\
& $0.5 \sigma$ & 100 & 0 & 100 & 100 \\
\hline
\end{tabular}



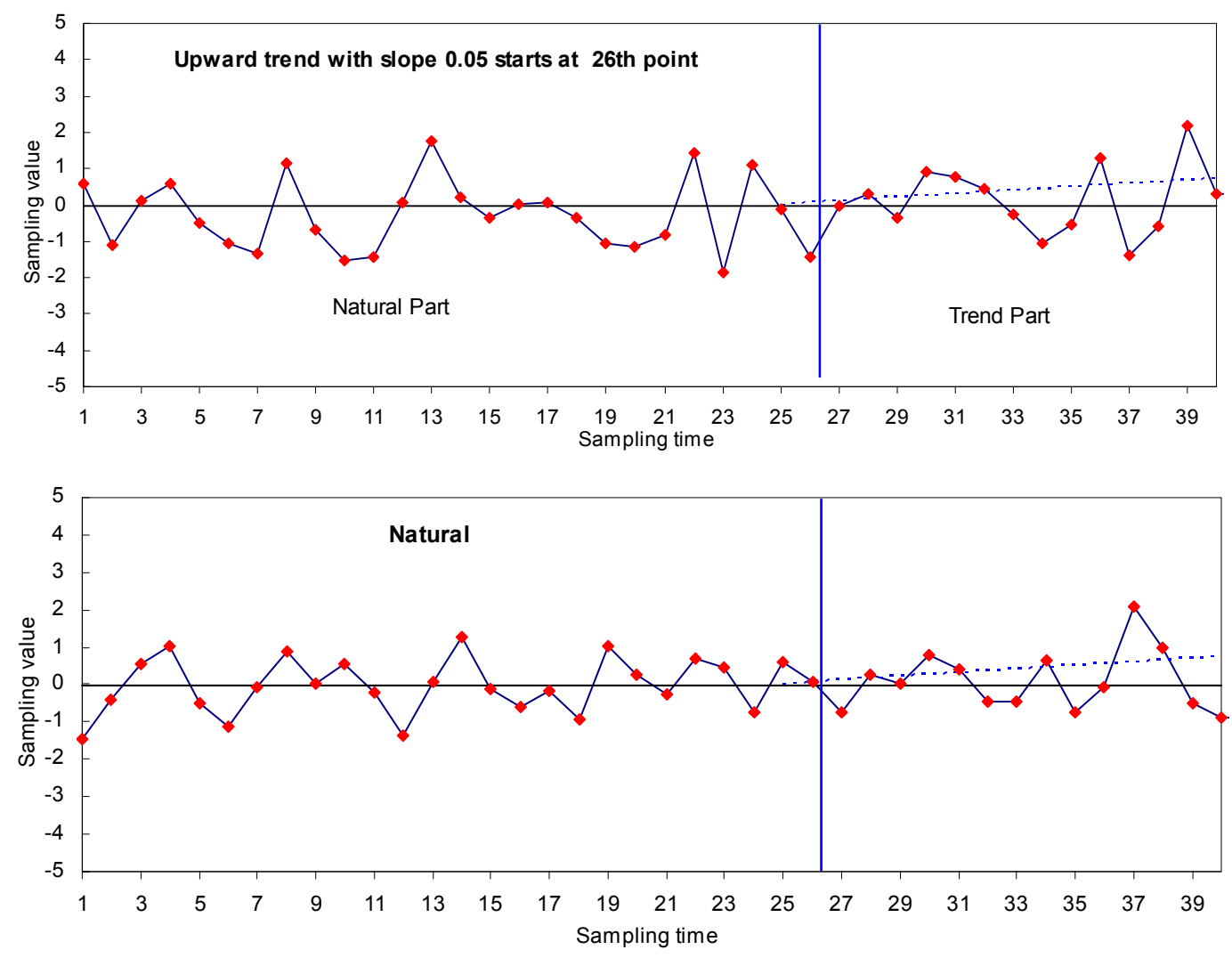

Figure 9. The similarity between trend pattern and natural at small slopes

Chakraborty [27] developed two feature-based approaches using heuristics and artificial neural network, which are capable of recognizing eight control chart patterns. They compared the results of the neural network with a heuristic approach and stated that the neural network results were better than heuristic results. Al-assaf [32] used the probability of success to compare between three approaches (DC, MRWA, and MRWA + DSC) to detect and classify the control chart unnatural patterns, his best results was obtained by using MRWA + DSC, so these results was used in the comparison. Guh [19] developed a hybrid learning-based model for on-line detection and analysis of control chart patterns; he trained a network to recognize eight control chart patterns and integrated this network in an algorithm to make it capable for on-line control chart analysis. In his work, the neural network testing results were reported based on the probability of success. Table 5 summarizes the comparison with their results.

The comparisons indicate that the trained network in this study is comparable if not superior. It has a good uniformly identification performance with the three basic control chart patterns. This proves that changing the network structure and using a compatible training algorithm with the network structure has a great effect on the
Table 5. Results comparison based on the percentage of success with the other authors

\begin{tabular}{ccccc}
\hline Pattern & $\begin{array}{c}\text { Proposed } \\
\text { ANN }\end{array}$ & $\begin{array}{c}\text { Al-Assaf } \\
(2004)\end{array}$ & $\begin{array}{c}\text { Guh } \\
(2005)\end{array}$ & $\begin{array}{c}\text { Gauri and } \\
\text { Chakraborty } \\
(2006)\end{array}$ \\
\hline Natural & 98.2 & 88.60 & 90.59 & 94.87 \\
$\begin{array}{c}\text { Upward } \\
\text { shift } \\
\begin{array}{c}\text { Upward } \\
\text { trend }\end{array}\end{array}$ & 100 & 93.20 & 93.33 & 93.40 \\
\hline
\end{tabular}

network performance.

\section{Conclusions}

This paper investigates a new approach to train a neural network to detect and identify the basic three control chart patterns natural, upward shift, and upward trend in addition to the traditional identification of runs. Instead of using a large training data set only small one can be used to do the job. Using a smaller training data set will make the network training convergence easier and using smaller set of patterns parameters in the training will eliminate the network to confusion and misclassification. Also a new network structure and a compatible training algorithm were suggested to make the network perform effectively. The results show that network can perform effectively and the percentage of suc- 
cess to identify a wide range of patterns is high and comparable if not superior to the previous reported results. This proves that changing the network structure and using a compatible training algorithm with the network structure has a great effect on the network performance.

\section{REFERENCES}

[1] R.-S. Guh, F. Zorriassatine, J. D. T. Tannock, and C. O' Brien, "On-line control chart pattern detection and discrimination: A neural network approach," Artificial Intelligence in Engineering, Vol. 13, pp. 413-425, 1999.

[2] T. L. Lucy-Bouler, "Using autocorrelations, CUSUMs and runs rules for control chart pattern recognition: An expert system approach," PhD dissertation, University of Alabama, Tuscaloosa, 1991.

[3] C.-S. Cheng and N. F. Hubele, "Design of a knowledgebased expert system for statistical process control," Computers and Industrial Engineering, Vol. 22, No. 4, pp. 501-517, 1992.

[4] J. A. Swift and J. H. Mize, "Out-of-control pattern recognition and analysis for quality control charts using Lisp-based systems," Computers and Industrial Engineering, Vol. 28, No. 1, pp. 81-91, 1995.

[5] E. L. Grant and R. S. Leavenworth, "Statistical quality control," 7th Edition, McGraw-Hill, New York, 1996.

[6] L. S. Nelson, "The Shewhart control chart: Tests for special causes," Journal of Quality Technology, Vol. 16, pp. 237-239, 1984.

[7] Western Electric, "Statistical quality control handbook," AT\&T, Princeton, 1956.

[8] D. C. Montgomery, "Introduction to statistical quality control," 3rd Edition, Wiley, New York, 1996.

[9] A. E. Smith, "X-Bar and R control chart interpretation using neural computing," International Journal of Production Research, Vol. 32, pp. 309-320, 1994.

[10] Y. Guo and K. J. Dooley, "Identification of change structure in statistical process control," International Journal of Production Research, Vol. 30, pp. 1655-1669, 1992.

[11] G. A. Pugh, "Synthetic neural networks for process control," Computers and Industrial Engineering, Vol. 17, pp. 24-26, 1989.

[12] G. A. Pugh, "A comparison of neural networks to SPC charts," Computers and Industrial Engineering, Vol. 21, pp. 253-255, 1991.

[13] S. I. Chang and E. S. HO, "A two-stage neural network approach for process variance change detection and classification," International Journal of Production Research, Vol. 37, No. 7, pp. 1581-1599, 1999.

[14] S. I. Chang and E. S. Ho, "An integrated neural network approach for simultaneous monitoring of process mean and variance shifts a comparative study," International Journal of Production Research, Vol. 37, pp. 1881-1901, 1999.

[15] I. Dedeakayogullari and N. Burnak, "Determination of mean and/or variance shifts with artificial neural networks," International Journal of Production Research. Vol. 37, No. 10, pp. 2191-2200, 1999.

[16] C.-S. Cheng and H.-P. Cheng, "Identifying the source of variance shifts in the multivariate process using neural networks and support vector machines," Expert Systems with Applications, Vol. 35, No. 1-2, pp. 198-206, 2008.

[17] L.-H. Chena and T.-Y. Wang, "Artificial neural networks to classify mean shifts from multivariate $X^{2}$ chart signals," Computers \& Industrial Engineering, Vol. 47, pp. 195-205, 2004.

[18] R.-S. Guh and Y.-C. Hsieh, "A neural network based model for abnormal pattern recognition of control charts," Computers and Industrial Engineering, Vol. 36, pp. 97-108, 1999.

[19] R.-S. Guh, "A hybrid learning-based model for on-line detection and analysis of control chart patterns," Computers and Industrial Engineering, Vol. 49, pp. 35-62, 2005.

[20] M. B. Perry, J. K. Spoerre, and T. Velasco, "Control chart pattern recognition using back propagation artificial neural networks," International Journal of Production Research, Vol. 39, pp. 3399-3418, 2001.

[21] K. Assaleh and Y. Al-assaf "Features extraction and analysis for classifying causable patterns in control charts," Computers and Industrial Engineering, Vol. 49. pp. 168-181, 2005.

[22] A. Hassan, M. S. N. Baksh, A. M. Shaharoun, and H. Jamaluddin, "Improved SPC chart pattern recognition using statistical features," International Journal of Production Research, Vol. 41, No. 7, pp. 1587-1603, 2003.

[23] A. Hassan, M. S. N. Baksh, A. M. Shaharoun, and H. Jamaluddin, "Feature selection for SPC chart pattern recognition using fractional factorial experimental design," Intelligent Production Machines and System: 2nd I*IPROMS Virtual International Conference, In: D. T. Pham, E. E. Eldukhri, and A. J. Soroka Ed., Elsevier, pp. 442-447, 3-14 July 2006.

[24] T. Zan, R.-Y. Fei, and M. Wang, "Research on abnormal pattern recognition for control chart based on neural network," Beijing Gongye Daxue Xuebao, Journal of Beijing University of Technology, Vol. 32, No. 8, pp. 673-676, 2006.

[25] Z. Chen, S. Lu, and S. Lam, "A hybrid system for SPC concurrent pattern recognition," Advanced Engineering Informatics, Vol. 21, No. 3, pp. 303-310, 2007.

[26] C.-S. Cheng, "A multi-layer neural network model for detecting changes in the process mean," Computers and Industrial Engineering, Vol. 28, No. 1, pp. 51-61, 1995.

[27] S. K. Gauri and S. Chakraborty, "Feature-based recognition of control chart patterns," Computers and Industrial Engineering, Vol. 51, pp. 726-742, 2006.

[28] F. Zorriassatine and J. D. T. Tannock, "A review of neural networks for statistical process control," Journal of Intelligent Manufacturing, Vol. 9, pp. 209-224, 1998. 
[29] S. Sagiroglu, E. Besdok, and M. Erler, "Control chart pattern recognition using artificial neural networks," Turkish Journal of Electrical Engineering, Vol. 8, No. 2, pp. 137-147, 2000.

[30] R.-S. Guh, "Optimizing feedforward neural networks for control chart pattern recognition through genetic algorithms," International Journal of Pattern Recognition and Articial Intelligence, Vol. 18, No. 2, pp. 75-99.

[31] H. Demuth, M. Beale, and M. Hagan "Neural network toolbox user's guide," Math Works, Natick, MA, 2009.

[32] Y. Al-Assaf, "Recognition of control chart patterns using multi-resolution wavelets analysis and neural networks," Computers and Industrial Engineering, Vol. 47, pp. 17-29, 2004. 


\section{Appendix A}

Pattern generation detailed flowchart

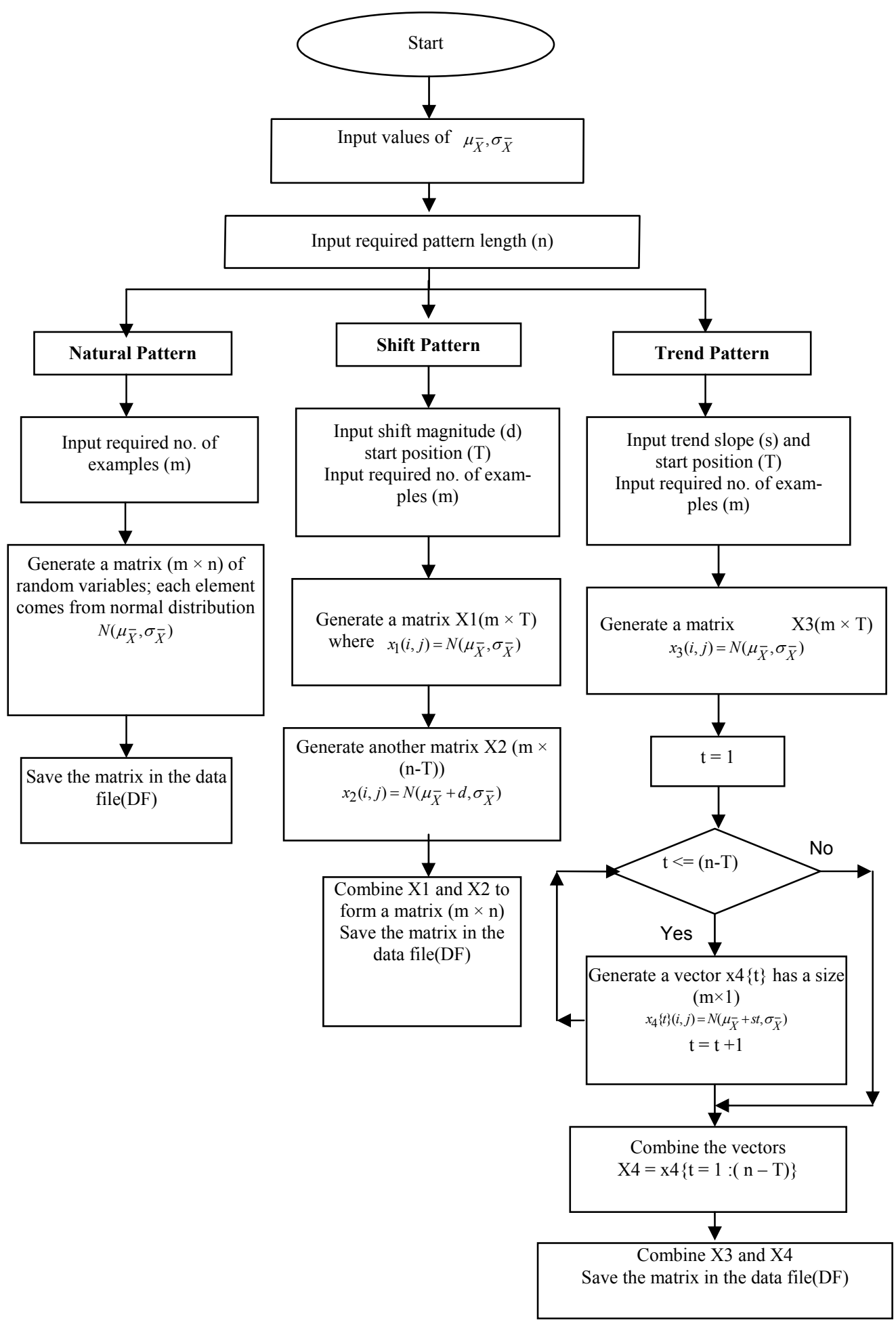

